\title{
DESTRUCTION OF MASSIVE FRAGMENTS IN PROTOSTELLAR DISKS AND CRYSTALLINE SILICATE PRODUCTION
}

\author{
EDUARD I. VOROBYOV ${ }^{1}$ \\ Institute for Computational Astrophysics, Saint Mary’s University, Halifax, B3H 3C3, Canada; vorobyov@ap.smu.ca \\ AND \\ Research Institute of Physics, Southern Federal University, Stachki 194, Rostov-on-Don, 344090, Russia \\ Received 2010 November 14; accepted 2011 January 15; published 2011 February 2
}

\begin{abstract}
We present a mechanism for the crystalline silicate production associated with the formation and subsequent destruction of massive fragments in young protostellar disks. The fragments form in the embedded phase of star formation via disk fragmentation at radial distances $\gtrsim 50-100 \mathrm{AU}$ and anneal small amorphous grains in their interior when the gas temperature exceeds the crystallization threshold of $\sim 800 \mathrm{~K}$. We demonstrate that fragments that form in the early embedded phase can be destroyed before they either form solid cores or vaporize dust grains, thus releasing the processed crystalline dust into various radial distances from sub-AU to hundred-AU scales. Two possible mechanisms for the destruction of fragments are the tidal disruption and photoevaporation as fragments migrate radially inward and approach the central star and also dispersal by tidal torques exerted by spiral arms. As a result, most of the crystalline dust concentrates to the disk inner regions and spiral arms, which are the likely sites of fragment destruction.
\end{abstract}

Key words: circumstellar matter - hydrodynamics - stars: formation - stars: protostars

Online-only material: color figures

\section{INTRODUCTION}

The embedded phase of low-mass star formation, starting from the formation of a disk and ending with the clearing of a parent cloud core, has recently drawn a renewed interest due to the possibility of planet formation in this phase. Indeed, circumstellar disks are most massive in the embedded phase (Andrews \& Williams 2005; Vorobyov 2009; Greaves \& Rice 2010) and numerical hydrodynamics simulations indicate that, under certain conditions, embedded disks can fragment and form either protoplanetary embryos or proto-brown dwarfs, especially in the disk outer regions where stellar irradiation and viscous heating are merciful (e.g., Durisen et al. 2007; Stamatellos \& Whitworth 2009; Boley 2009; Machida et al. 2010; Vorobyov \& Basu 2010b; Cha \& Nayakshin 2011).

While disk fragmentation in the embedded phase seems certainly feasible, the prospects for the survival of fragments and formation of gas giants or brown dwarfs on distances of order tens or hundreds AU are gloomy. Gravitational instability in the embedded phase is strong, fuelled with a continuing infall of gas from a parent cloud core, and resultant gravitational and tidal torques are rampant. As a consequence, part of the fragments are dispersed by the tidal torques exerted by the spiral arms (Vorobyov \& Basu 2010b; Boley et al. 2010). Others are quickly driven into the disk inner regions, and probably onto the star, due to the loss of angular momentum caused by the gravitational interaction with the trailing spiral arms (Vorobyov \& Basu 2006, 2010b; Cha \& Nayakshin 2011). Only those fragments that happen to form in the late embedded phase, when gravitational instability and associated torques are getting weaker, may open a gap in the disk and mature into gas giants on wide orbits but the probability for such a "fortunate" outcome is rather low (Vorobyov \& Basu 2010a).

Ironic as it may seem, but the destruction of fragments (hereafter planet/brown-dwarf embryos or simply embryos) may

\footnotetext{
1 Visiting Scientist, Department of Physics and Astronomy, The University of Western Ontario, London, ON N6A 3K7, Canada.
}

be as important for the disk and stellar evolution as their survival followed by massive giant planet or brown dwarf formation. Embryos that are driven into the disk inner regions may form solid cores in their interior via dust sedimentation or dense and compact atomic hydrogen cores via dissociation of molecular hydrogen. If one or both of those processes take place before the embryo is destroyed on its approach to the central star, a planet can emerge on orbit of order several AU or less (Nayakshin 2010a; Boley et al. 2010). If both processes fail, then the release of gravitational energy from the accreted embryo can trigger a luminosity outburst similar in magnitude those of FU Orionis or EX Lupi family (Vorobyov \& Basu 2006, 2010b). In addition, short but intense bursts of accretion onto the star caused by the consumption of embryos can lower the lithium abundance in young solar-type stars (Baraffe \& Chabrier 2010), explain the luminosity spread in H-R diagrams of 1 Myr old star clusters (Baraffe et al. 2009), and resolve the long-standing luminosity problem (Dunham et al. 2010).

On the other hand, embryos are sites of accelerated dust growth and high-temperature processing (Boss 1998; Boss et al. 2002; Boley et al. 2010; Nayakshin 2010a, 2010b; Nayakshin et al. 2011) and their in situ destruction by tidal torques may release processed dust directly into the disk outer regions.

Whichever mechanism of embryo destruction is realized in nature, the consequences of this process are far-stretching. In this Letter, we report another interesting by-product of the embryo dispersal - the enrichment of embedded circumstellar disks with crystalline silicates formed in the depths of massive embryos via thermal annealing of amorphous dust grains. We use numerical hydrodynamics simulations of the disk formation and long-term evolution complemented with equations describing the transformation of micron-sized amorphous dust grains into the silicate form. We show that part of the crystallinesilicate-bearing embryos are destroyed before they vaporize dust grains in their interiors or form solid cores via dust sedimentation, thus enriching the gas disk with crystalline silicates on radial distances from sub-AU to hundred-AU scales. 


\section{SOURCES OF CRYSTALLINE SILICATES IN CIRCUMSTELLAR DISKS}

Circumstellar disks originate during the gravitational collapse of rotating prestellar cloud cores. The degree in crystallinity of silicates in prestellar cores is similar to that of the interstellar medium, which has an upper limit of $0.2 \% \pm 0.2 \%$ in the direction of the Galactic center (Kember et al. 2004). On the other hand, the crystalline-silicate fraction in the inner regions of circumstellar disks around young stellar objects (YSOs) can vary in wide limits from essentially none to almost $100 \%$ (Watson et al. 2009), implying in situ production of crystalline silicates in at least some YSOs. Two mechanisms for dust crystallization have been put forward.

1. Evaporation of the original, amorphous dust grains followed by re-condensation under conditions of high temperature and density (e.g., Grossman 1972).

2. Thermal annealing of the amorphous grains at temperatures (800-1300) K, somewhat below their vaporization point, via viscous heating (e.g., Gail 2001), shock wave heating (Harker \& Desch 2002), or disk surface heating during EX-Lupi-like outbursts (Ábrahám et al. 2009).

Both mechanisms are thought to work mostly in the inner 1 AU from the star (except for shock heating that may operate at somewhat larger radial distances $r \lesssim 10 \mathrm{AU}$ ) and some means of outward radial transport of crystallized dust is necessary to account for the non-zero crystalline-silicate fraction in comets at distances of order tens of AU (e.g., Gail 2001; Bockelée-Morvan et al. 2002; Ciesla 2007).

\section{MODEL DESCRIPTION AND INITIAL CONDITIONS}

Our numerical model is explained in detail in Vorobyov \& Basu (2010b). Here, we provide only a brief overview and describe modifications done to the numerical code. We compute the gravitational collapse of rotating cloud cores in the thin-disk approximation and start our simulations from the pre-stellar phase, advance through the disk formation and early evolution phase, and terminate with almost a complete clearing of the natal core. The following physical effects are taken into account in our model: stellar irradiation, background irradiation with temperature $T_{\mathrm{bg}}=10 \mathrm{~K}$, viscous and shock heating, radiative cooling from the disk surface, and disk self-gravity. Turbulent viscosity is parameterized using the usual $\alpha$-prescription. A spatially and temporally constant $\alpha$ is set to 0.005 .

In the current version, we have implemented a simple mechanism describing the transformation of amorphous dust grains into the crystalline form following the guidelines described in Dullemond et al. (2006). We assume that dust is passively transported with gas and neglect the dust radial drift caused by the dust-gas drag force. The latter simplification is of little consequence for the dynamics of dust grains with sizes $\lesssim 10 \mu \mathrm{m}$ on timescales of interest for the present paper, $\lesssim 0.5 \mathrm{Myr}$ (Takeuchi $\&$ Lin 2002). Therefore, our results are strictly applicable only to sufficiently small dust grains showing sharp features in the 5-35 $\mu \mathrm{m}$ band of the mid-infrared spectrum. In this simple scheme, two additional continuity equations for the surface density of amorphous and crystalline silicates, $\Sigma_{\text {a.s. }}$ and $\Sigma_{\text {c.s. }}$, respectively, are

$$
\frac{\partial \Sigma_{\text {a.s. }}}{\partial t}+\nabla_{p} \cdot\left(\Sigma_{\text {a.s. }} \boldsymbol{v}_{p}\right)=-S,
$$

$$
\frac{\partial \Sigma_{\text {c.s. }}}{\partial t}+\nabla_{p} \cdot\left(\Sigma_{\text {c.s. }} \boldsymbol{v}_{p}\right)=+S,
$$

where $\boldsymbol{v}_{p}=v_{r} \hat{\boldsymbol{r}}+v_{\phi} \hat{\boldsymbol{\phi}}$ is the gas velocity in the disk plane and $S=v_{\text {cr }} \Sigma_{\text {a.s. }}$. All dust grains in a collapsing cloud core are initially amorphous, they crystallize at a rate $v_{\text {cr }}$ provided by the Arrhenius formula

$$
v_{\mathrm{cr}}\left[\mathrm{s}^{-1}\right]=2 \times 10^{13} \exp \left(-\frac{T_{\mathrm{c}}}{T_{\mathrm{g}}}\right),
$$

where $T_{\mathrm{g}}$ is the gas midplane temperature, $2 \times 10^{13}$ is the lattice vibrational frequency, and $T_{\mathrm{c}}=38,100 \mathrm{~K}$ (Bockelée-Morvan et al. 2002). We assume that dust and gas are thermalized. The initial gas and dust radial surface density profiles and angular velocities are given by Equations (12) and (13) in Vorobyov $\&$ Basu (2010b) and the initial dust-to-gas ratio is 0.01 . The initial core mass is $M_{\text {core }}=0.78 M_{\odot}$ and the ratio of rotational to gravitational energy of the core is $\beta=8.8 \times 10^{-3}$. For simplicity, we also assume that all dust is made of silicate grains.

A solution of the time-dependent part of Equation (2) (excluding advection terms) demonstrates that it takes about $40 \mathrm{yr}$ to completely transform amorphous dust into the crystalline form at $T_{\mathrm{g}}=800 \mathrm{~K}$. The corresponding timescale for $T_{\mathrm{g}}=900 \mathrm{~K}$ is $0.1 \mathrm{yr}$, while for $T_{\mathrm{g}}=700 \mathrm{~K}$ the crystallization timescale is $20,000 \mathrm{yr}$. Obviously, the latter value is much longer than the typical orbital period and crystallization temperatures of $T_{\mathrm{cr}}=800-900 \mathrm{~K}$ are most relevant to circumstellar disks. Laboratory experiments tend to yield even higher $T_{\text {cr }} \gtrsim 1000 \mathrm{~K}$ (Hallenbeck et al. 2000), possibly due to the same timescale arguments.

If temperature exceeds the evaporation threshold for dust grains $T_{\text {evap }} \approx 1400 \mathrm{~K}$, we set both $\Sigma_{\text {c.s. }}$ and $\Sigma_{\text {a.s. }}$ to zero in the corresponding computational cell. The exact value of $T_{\text {evap }}$ is density dependent and is calculated from the opacity tables of Bell \& Lin (1994).

Equations (1) and (2) are solved simultaneously with equations of hydrodynamics describing the formation and evolution of circumstellar disks (Equations (1)-(3) in Vorobyov \& Basu $2010 b$ ). We employ the method of finite differences with a timeexplicit, operator-split solution procedure in polar coordinates $(r, \phi)$ on a numerical grid with $512 \times 512$ grid zones. The advection part of Equations (1) and (2) (i.e., with $S$ set to zero) is solved using a piecewise parabolic advection scheme. The time update of $\Sigma_{\text {a.s. }}$ and $\Sigma_{\text {c.s. }}$ due to the source/sink term $S$ is done using an (unconditionally stable) backward difference scheme supplemented with a subcycling procedure to guarantee accuracy, wherein the change in both $\Sigma_{\text {a.s. }}$ and $\Sigma_{\text {c.s. }}$ over one global hydrodynamical time step is limited to $10 \%$. If this condition is violated, local subcycling with smaller time steps is invoked until the desired accuracy is reached.

The radial points in our numerical grid are logarithmically spaced. The innermost grid point is located at the position of the sink cell $r_{\mathrm{sc}}=6 \mathrm{AU}$ and the size of the first adjacent cell is $0.08 \mathrm{AU}$. This corresponds to the radial resolution of $\Delta r=$ $1.3 \mathrm{AU}$ at $100 \mathrm{AU}$. We impose a free outflow boundary condition so that the matter is allowed to freely flow through the sink cell to form a central star plus some dynamically inactive inner region.

\section{RESULTS}

The top row in Figure 1 presents the gas surface density in the inner several hundred $\mathrm{AU}$ at three distinct evolution times since the formation of the central star. The middle and bottom rows show the corresponding gas midplane temperature and 

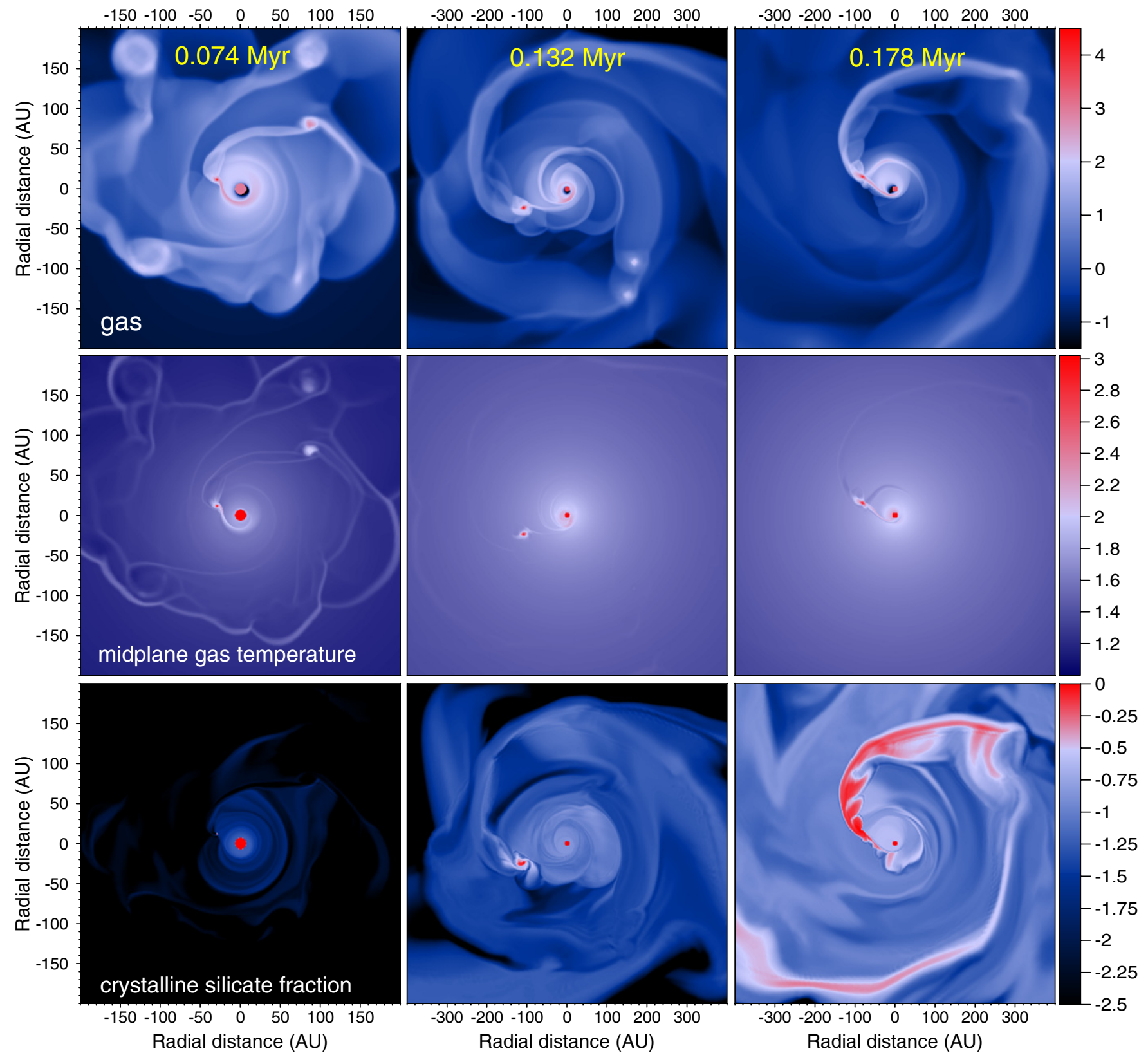

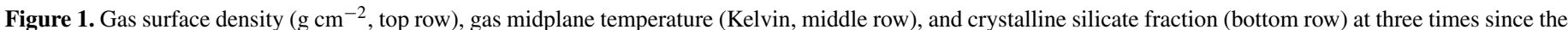
formation of the central star: $t=0.074 \mathrm{Myr}$ (left column), $t=0.132 \mathrm{Myr}$ (middle column), and $t=0.178 \mathrm{Myr}$ (right column).

(A color version of this figure is available in the online journal.)

crystalline silicate fraction $\xi_{\text {c.s. }}=M_{\text {c.s. }} /\left(M_{\text {c.s. }}+M_{\text {a.s. }}\right)$, respectively, where $M_{\text {c.s }}$ and $M_{\text {a.s. }}$ are crystalline and amorphous silicate masses. The red circle in the coordinate center represents schematically the central star plus sink cell. The three time snapshots in Figure 1 are chosen specifically to illustrate disk fragmentation and embryo formation (red dots at 50-100 AU). We note that each snapshot shows in fact different embryos-their inward migration and/or tidal destruction timescales are of order several orbital periods $\left(T_{\text {orb }}=700 \mathrm{yr}\right.$ for $M_{*}=0.25 M_{\odot}$, $r=50 \mathrm{AU}, t=0.074 \mathrm{Myr}$, and $T_{\mathrm{orb}}=1600 \mathrm{yr}$ for $M_{*}=0.4$, $r=100 \mathrm{AU}, t=0.178 \mathrm{Myr})$, which are considerably shorter than the time span between the images $\left(\sim 5 \times 10^{4} \mathrm{yr}\right)$.

We find that the gas temperature $T_{\mathrm{g}}$ in the interiors of massive embryos can exceed $800 \mathrm{~K}$ (see the middle row in Figure 1), necessary for the thermal annealing of amorphous dust grains to commence. The prospects for disk enrichment with crystalline silicates stored in these embryos will depend on characteristic timescales for inward migration and/or tidal destruction of embryos ( $\left.\tau_{\text {dest }}\right)$, dust sedimentation and solid core formation in the embryo interiors $\left(\tau_{\mathrm{sed}}\right)$, and dust vaporization at $T_{\mathrm{g}}>T_{\text {evap }}\left(\tau_{\text {evap }}\right)$. If $\tau_{\text {dest }}$ is smaller than both $\tau_{\text {sed }}$ and $\tau_{\text {evap }}$, then all processed dust will be released into the disk at various radial distances, depending on which destruction mechanism prevails. As current numerical simulations indicate, embryos may be destroyed by tidal torques from spiral arms, preferentially releasing the crystalline silicates to large radial distances where these embryos form. Alternatively, embryos may be photoevaporated and torn apart by stellar irradiation and tidal torques as they migrate radially inward and approach the central star. In this case, part of the processed dust will be 


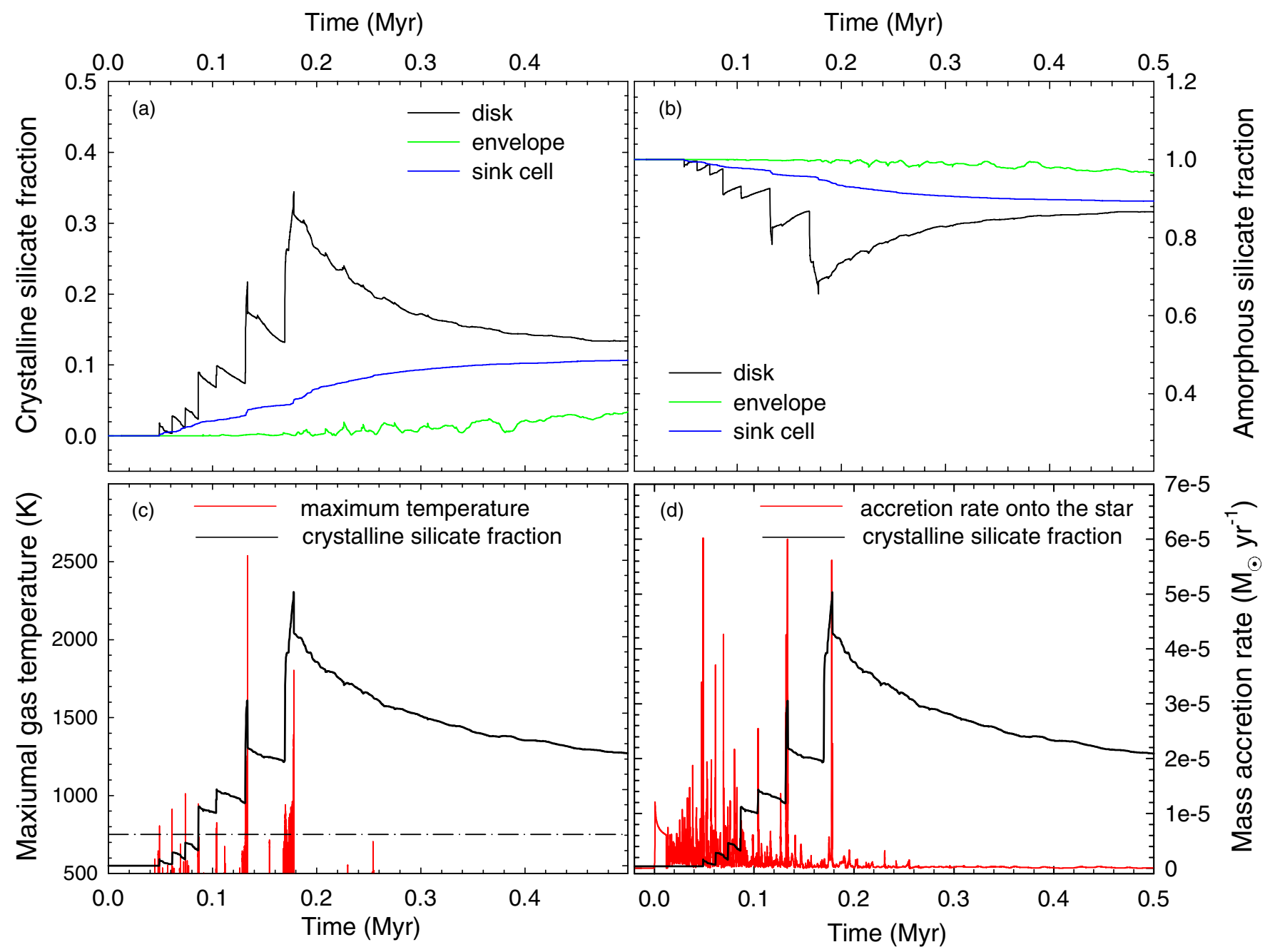

Figure 2. Crystalline (panel (a)) and amorphous (panel (b)) silicate fractions in the disk (black), envelope (green), and star+sink cell (blue) as a function of time passed since the formation of the central star. Panel (c) shows the maximum temperature in the disk (red) and crystalline silicate fraction in the disk (black), while panel (d) also shows the mass accretion rate onto the star (red).

(A color version of this figure is available in the online journal.)

brought into the disk inner few AU (through the sink cell) and probably onto the star and part will be pushed to larger disk radii during a transient episode of disk expansion (caused by the conservation of angular momentum) following the embryo tidal disruption. It is thus feasible to deposit crystalline silicates to various orbital distances starting from sub-AU scales and up to several hundred $\mathrm{AU}$, accounting for the crystalline silicate features observed in the spectra of the solar system comets. This process of crystalline silicate enrichment is illustrated in the bottom row of Figure 1 -there is a notable increase in $\xi_{\text {c.s. }}$ with time. Crystalline silicates concentrate in the disk inner regions and at/near spiral arms, which are likely sites of embryo formation and destruction. This implies that the abundance of crystalline silicates may increase with radius, at least in the early disk evolution, if most of the embryos are tidally destroyed in the disk outer regions rather than driven into the star. If such an increase is observationally confirmed, it could become a hallmark for the proposed process.

On the other hand, if $\tau_{\text {dest }}$ greater than either $\tau_{\text {sed }}$ or $\tau_{\text {evap }}$, then most of the processed crystalline dust will be either locked up in solid terrestrial-like cores or vaporized. The latter mechanism is implemented in the current numerical model, while the effect of the former is discussed in more detail later in the text.
To better illustrate the process of disk enrichment with crystalline silicates, Figure 2 presents various model characteristics as a function of time. In particular, the crystalline silicate fraction $\xi_{\text {c.s. }}$ and amorphous silicate fraction $\xi_{\text {a.s. }}=M_{\text {a.s. }} /\left(M_{\text {c.s. }}+M_{\text {a.s. }}\right)$ are plotted in panels (a) and (b), respectively. More specifically, black, green, and blue lines depict the corresponding fractions in the disk ( $\xi_{\text {c.s. }}^{\text {disk }}$ and $\left.\xi_{\text {a.s. }}^{\text {disk }}\right)$, envelope ( $\xi_{\text {c.s. }}^{\text {env }}$ and $\xi_{\text {a.s. }}^{\text {env }}$ ), and sink cell $\left(\xi_{\text {c.s. }}^{\text {sc }}\right.$ and $\left.\xi_{a . s .}^{\text {sc }}\right)$, respectively. We distinguish between the disk and envelope using a scheme based on the typical transitional gas surface density and radial gas velocity field explained in detail in Vorobyov (2010). We note that $\xi_{\text {c.s. }}^{\text {disk }}$ and $\xi_{\text {a.s. }}^{\text {disk }}$ are in fact crystalline and amorphous silicate fractions stored in both the disk and the embryos. Panel (c) shows the crystalline silicate fraction in the disk (black line) and maximum midplane gas temperature $T_{\mathrm{g}}^{\max }$ (red line), whereas panel (d) presents the mass accretion rate onto the star $\dot{M}$ (red line) and the crystalline silicate fraction in the disk (black line).

There are seven episodes of crystalline silicate production in the disk manifested by a sharp increase in $\xi_{c . s .}^{\text {disk }}$, all associated with the formation of embryos massive and hot enough to crystallize dust in their interior. Episodes 4, 6, and 7 (from left to right) correspond to the formation of embryos shown in the left, middle, and right columns of Figure 1. From panel 
(c) it is evident that $\xi_{\text {c.s. }}^{\text {disk }}$ increases sharply each time the maximum gas temperature $T_{\mathrm{g}}^{\max }$ exceeds the crystallization temperature $T_{\mathrm{cr}} \approx 800 \mathrm{~K}$ (horizontal dash-dotted line). After each such episode, prolonged periods of gradual decline in $\xi_{\mathrm{c} . \mathrm{s}}^{\text {disk }}$ follow, which are caused by dilution of the disk material with pristine amorphous dust accreted onto the disk from the infalling envelope.

In the last two episodes, $T_{\mathrm{g}}^{\max }$ goes above the dust evaporation temperature $T_{\text {evap }}$ and this leads to an immediate sharp drop in $\xi_{\text {c.s. }}^{\text {disk }}$. This exemplifies cases with $\tau_{\text {evap }}<\tau_{\text {dest }}$. The dust evaporation timescale can be approximated (excluding factors of unity) as (Nayakshin 2010a)

$$
\tau_{\text {evap }}=1.5 \times 10^{4}\left(\frac{M_{\mathrm{emb}}}{10 M_{\mathrm{J}}}\right)^{-2} \mathrm{yr} .
$$

For the typical embryo masses of $M_{\mathrm{emb}}=5-20 M_{\mathrm{J}}$, the corresponding $\tau_{\text {evap }}$ lies in the 4000-60,000 yr range. In fact, this is a lower estimate simply because embryos may experience several contraction and expansion episodes before actually reaching $T_{\text {evap }}$. Embryos that form in the early disk evolution are of lower mass and closer to the star than those that form later in the embedded phase (because disk grows in mass and size with time). As a consequence, first embryos take longer time to evaporate dust and shorter time to migrate into the star, an effect found in our numerical simulations and also noted by Cha \& Nayakshin (2011). For these first embryos, migration timescales are just several orbital periods $\left(T_{\text {orb }}=700\right.$ $1600 \mathrm{yr}$ ) and are often shorter than $\tau_{\text {evap. }}$. As time passes and more massive embryos on wider orbits start to form, the migration/ destruction timescale may exceed that of dust evaporation, as indeed occurred during the last two episodes of crystalline dust production.

Migration of embryos into the disk inner regions and through the sink cell, as manifested by strong $\left(\gtrsim 5 \times 10^{-5} M_{\odot} \mathrm{yr}^{-1}\right)$ mass accretion bursts, also contributes to the decrease in $\xi_{\text {c.s. }}^{\text {disk }}$ but leads to an increase in $\xi_{\text {c.s. }}^{\text {sc }}$. Note that episodes 3 and 4 are not associated with strong mass accretion bursts, indicating that the corresponding embryos are dispersed by tidal torques exerted by spiral arms, before they actually fall through the sink cell onto the star. The process of disk fragmentation diminishes after $t \approx 0.2 \mathrm{Myr}$, no embryos survive beyond this time, and the subsequent evolution shows a gradual decline in $\xi_{\text {c.s. }}^{\text {disk }}$. From this moment on, $\xi_{\mathrm{c} . \mathrm{s} \text {. }}^{\text {disk }}$ represents the crystalline silicate fraction in the disk only.

We do not take into account a possible formation of solid cores (and associated reduction in disk enrichment with crystalline dust) but we can estimate its efficiency based on the work of Nayakshin (2010a). For $M_{\mathrm{emb}}=3-10 M_{\mathrm{J}}$, Nayakshin found $\tau_{\text {sed }} \approx(4-6) \times 10^{3}$ yr. This is comparable to or less than $\tau_{\text {evap }} \gtrsim(4-16) \times 10^{3} \mathrm{yr}$, which implies that solid cores may form before dust is vaporized. At the same time, $\tau_{\text {sed }}$ is comparable to or longer than $\tau_{\text {dest }}=$ a few $\times T_{\text {orb }} \approx$ a few $\times 10^{3} \mathrm{yr}$, certainly for those embryos that form first in the early embedded phase and are characterized by shortest migration timescales. This simple timescale analysis suggests that the solid core formation is feasible and may reduce the efficiency of crystalline dust enrichment but is unlikely to shut down the proposed mechanism completely. The latest numerical hydrodynamics simulations seem to confirm that the solid core formation is not expected to work for every embryo in the disk (Cha \& Nayakshin 2011).

\section{SUMMARY}

Thermal annealing of amorphous dust grains in the hot interiors of massive fragments (or planet/brown dwarf embryos) forming in protostellar disks during the early embedded stages of stellar evolution, followed by subsequent dispersal of the fragments, is a promising gateway for the production of crystalline silicates. This mechanism can release processed dust to various radial distances from (sub-)AU scales, if fragments are destroyed on their approach to the central star, to hundred-AU scales, if they are dispersed by tidal torques exerted by spiral arms. More studies, including the effect of dust growth and solid core formation, are needed to further explore the efficiency of this mechanism.

We have run another eight models with $\beta=(3.2-12) \times 10^{-3}$ and $M_{\text {core }}=0.3-1.25 M_{\odot}$. The net result is that the dust processing is correlated with disk fragmentation and models that do not form embryos (e.g., models with low $M_{\text {core }}$ and $\beta$ and consequently with disks of low mass and size) show little crystalline silicates, reinforcing our conclusions.

We stress that our proposed mechanism can coexist with other crystallization mechanisms, localized to the inner few AU, and complements them by providing a direct source of crystalline silicates at large distances. Some outward transport may still take place and account for the fact that comet Wild 2 has crystalline silicates with the chemical and isotopic composition similar to that of the chondritic meteorites originated in the terrestrial planet region (McKeegan et al. 2006). Conversely, chondritic meteorites may have formed in the atmospheres of protoplanetary embryos via dust sedimentation and released into the inner few $\mathrm{AU}$ when the embryos were dispersed by tidal torques near the young protosun. The apparent presence of refractory calcium aluminum inclusions in the cometary material does not invalidate this scenario since temperatures in at least some of the embryos can exceed $1300 \mathrm{~K}$ (panel (c) in Figure 2). We do not take into account possible opacity dependence on the composition and properties of dust grains due to the complexity of physics involved. Nevertheless, dust growth and crystallization are likely to decrease opacities (Mennella et al. 1998; D'Alessio et al. 2001), thus enhancing disk fragmentation and crystalline silicate production.

The author is grateful to the anonymous referee for very useful comments and to Professor Shantanu Basu for hospitality. Support from an ACEnet Fellowship is gratefully acknowledged. Numerical simulations were done on the Atlantic Computational Excellence Network (ACEnet). This project was also supported by RFBR grant 10-02-00278 and by the Ministry of Education grant RNP 2.1.1/1937.

\section{REFERENCES}

Ábrahám, P., et al. 2009, Nature, 459, 224

Andrews, S. M., \& Williams, J. P. 2005, ApJ, 631, 1134

Baraffe, I., \& Chabrier, G. 2010, A\&A, 521, 44

Baraffe, I., Chabrier, G., \& Gallardo, J. 2009, ApJ, 702, L27

Bell, K. R., \& Lin, D. N. C. 1994, ApJ, 427, 987

Bockelée-Morvan, D., Gautier, D., Hersan, F., Huré, J.-M., \& Robert, F. 2002, A\&A, 384, 1107

Boley, A. C. 2009, ApJ, 695, L53

Boley, A. C., Hayfield, T., Mayer, L., \& Durisen, R. H. 2010, Icarus, 207, 509

Boss, A. P. 1998, ApJ, 503, 923

Boss, A. P., Wetherill, G. W., \& Haghighipour, N. 2002, Icarus, 156, 291

Cha, S.-H., \& Nayakshin, S. 2011, MNRAS, in press (arXiv:1010.1489)

Ciesla, F. 2007, Science, 318, 613 
D’Alessio, P., Calvet, N., \& Hartmann, L. 2001, ApJ, 553, 321

Dunham, M. M., Evans, N. J., II, Terebey, S., Dullemond, C. P., \& Young, C. H. 2010, ApJ, 710, 470

Dullemond, C. P., Apai, D., \& Walch, S. 2006, ApJ, 640, L67

Durisen, R. H., Boss, A. P., Mayer, L., Nelson, A. F., Quinn, T., \& Rice, W. K.

M. 2007, in Protostars and Planets V, ed. B. Reipurth, D. Jewitt, \& K. Keil

(Tucson, AZ: Univ. Arizona Press), 607

Gail, H.-P. 2001, A\&A, 378, 192

Greaves, J. S., \& Rice, W. K. M. 2010, MNRAS, 407, 1981

Grossman, L. 1972, Geochim. Cosmochim. Acta, 36, 597

Hallenbeck, S. L., Nuth, J. A., III., \& Nelson, R. N. 2000, ApJ, 535, 247

Harker, D. E., \& Desch, S. J. 2002, ApJ, 565, L109

Kemper, F., Vriend, W. J., \& Tielens, G. G. M. 2004, ApJ, 609, 826

Machida, M. N., Inutsuka, S., \& Matsumoto, T. 2010, ApJ, 724, 1006
McKeegan, K. D., et al. 2006, Science, 314, 1724

Mennella, V., Brucato, J. R., Colangeli, L., Palumbo, P., Rotundi, A., \& Bussoletti, E. 1998, ApJ, 496, 1058

Nayakshin, S. 2010a, MNRAS, 408, 36

Nayakshin, S. 2010b, MNRAS, 408, 2381

Nayakshin, S., Cha, S.-H., \& Bridges, J. 2011, arXiv: 1101.1035

Stamatellos, D., \& Whitworth, A. P. 2009, MNRAS, 392, 413

Takeuchi, T., \& Lin, D. N. C. 2002, ApJ, 581, 1344

Vorobyov, E. I. 2009, ApJ, 692, 1609

Vorobyov, E. I. 2010, ApJ, 713, 1059

Vorobyov, E. I., \& Basu, S. 2006, ApJ, 650, 956

Vorobyov, E. I., \& Basu, S. 2010a, ApJ, 714, L133

Vorobyov, E. I., \& Basu, S. 2010b, ApJ, 719, 1896

Watson, D. M., et al. 2009, ApJSS, 180, 84 